\title{
Development of a Novel Bioactive Functionally Guided Tissue Graded Membrane for Periodontal Lesions
}

\author{
S. B. Qasim ${ }^{1}$, R. Delaine-Smith ${ }^{1}$, A. Rawlinson ${ }^{2}$, I. U. Rehman ${ }^{1}$ \\ ${ }^{1}$ Materials Science and Engineering Department, Kroto Research Institute, University of Sheffield. \\ ${ }^{2}$ Academic Unit of Restorative Dentistry, Charles Clifford Dental School, University of Sheffield.
}

\begin{abstract}
Periodontal disorders are chronic inflammatory condition of the supporting structures of the tooth. Guided tissue regeneration (GTR) membranes have been used in the management of destructive forms of periodontal disease as a means of aiding regeneration of lost supporting tissues, such as alveolar bone, cementum, gingiva and periodontal ligament. In this study, individual layers of a functionally graded bioactive composite membrane were fabricated using the techniques of solvent casting, freeze gelation and electrospinning by incorporating hydroxyapatite (HA) alongside Chitosan and using acetic acid as the primary solvent. The techniques were simple, time /energy efficient and reproducible resulting in novel non-porous and porous scaffolds with potential for use in periodontal tissue regeneration. Scaffolds were characterised in terms of ultra-structure morphology by scanning electron microscopy, physiochemical properties, water uptake, and degradation by weight loss, mechanical properties and biocompatibility. Microscopy revealed porous features and presence of hydroxyapatite was confirmed with Fourier transform infrared (FTIR) spectroscopy. Morphology of the pores varied with different solvents, which were affected by addition of HA. Biocompatibility studies were carried out using a human osteosarcoma cell line and human embryonic stem cell-derived mesenchymal progenitor cells. All tested scaffolds showed increasing cell viability over the tested culture period. In conclusion, solvent casting, freeze gelation and electrospinning were successfully used to fabricate scaffolds with different morphology and porosity. Future work will focus on incorporation of drug into the core layer and combining the surface and core layers to form a spatially designed functionally graded biodegradable membrane with the potential of being used for periodontal tissue regeneration.
\end{abstract}

Keywords Chitosan; Guided Tissue Regeneration; Hydroxyapatite; Periodontal

\section{INTRODUCTION}

Periodontitis is a globally prevalent chronic inflammatory disease of the soft and hard tissues supporting teeth and a major cause of tooth loss. It has now become more common than the cold and the flu. The National Health Service spends around $\mathrm{f2.78}$ billion in the UK towards periodontal treatments [1]. Data from the National Institute of Health has revealed that more than $90 \%$ of adult population have moderate levels of periodontal disease [2]. Beyond its implication of oral health, it has become more evident that during the last decade periodontal disease may also have an impact on systemic health as well. Major advances in periodontal treatment strategies have resulted in paradigm shifts in tissue engineering and regenerative medicine. Out of the very few possibilities of preventing tooth loss, one of the last resorts available is the use of a guided tissue regenerative (GTR) membrane. Although current GTR membranes have shown promising results, there are some critical limitations preventing complete regeneration. An ideal GTR membrane will exhibit properties allowing tissue integration acting as a space maintainer for new tissue growth; provide stability to the underlying clot acting as a barrier; and ease of handling. The limitations to all currently available GTR membranes are relatively high variation, low predictability of clinical attachment, and bone growth. Others have many structural, mechanical and biofunctional limitations as well. In order to accomplish predictable tissue regeneration it is pivotal to stimulate precursor cells by adhesion molecules and messengers which should be capable of physiologically promoting cell and tissue adhesion [3].
The aim of the project is to fabricate a functionally graded (Fig. 1), spatially designed bioactive degradable membrane that could be used in periodontal regeneration.

\section{Materials AND Methodology}

Chitosan $(\mathrm{CH})$ has been used as the core polymer for fabricating this functionally graded structure. In all trilayered compartments fabricated, $\mathrm{CH}$ has been dissolved in $0.2 \mathrm{M}$ acetic acid. The bioactive ingredient utilised is hydroxyapatite (HA) (Plasma Biotal R). A non-porous surface layer has been made by solvent casting $\mathrm{CH}$ and $\mathrm{HA}$ with different ratios of HA. A core layer has been fabricated by using a time-saving energy-efficient novel method of freeze gelation. The bottom surface layer has been synthesised by electrospinning $\mathrm{CH}$ to obtain an oriented and highly aligned fibre orientation. Morphological characterisation of each layer was carried out by scanning electron microscopy (SEM). Chemical structure was evaluated by Fourier transform infrared (FTIR) spectroscopy - mechanical strength was observed by tensile tests performed at both wet and dry conditions. Swelling ratio and weight loss analysis was also performed. Biocompatibility of fabricated scaffolds was assessed with Alamar blue using human osteosarcoma and mesenchymal progenitor cell line. Calcium and collagen deposition was assessed using Alizarin red and Sirius red staining.

\section{RESULTS AND DISCUSSION}

Functionally graded layers were successfully prepared, the SEM (Fig. 2) images show different morphology of the top and bottom surface achieved, with the bottom surface 
showing dominance of HA particles, freeze gelated scaffolds showing porous structure, and electrospun fibres showing random and aligned orientation. FTIR spectral (Fig. 3) data of the freeze gelated scaffolds depicts successful incorporation of $\mathrm{HA}$ within $\mathrm{CH}$, presence of the functional group $(-\mathrm{OH})$ of $\mathrm{HA}$ at $3568 \mathrm{~cm}^{-1}$, and vibration modes of Phosphate $\left(\mathrm{PO}_{4}{ }^{-3}\right) v_{1}$ at $962 \mathrm{~cm}^{-1}$ point toward the presence of $\mathrm{HA}$ within the $\mathrm{CH}$ matrix, possibly forming covalent or ionic interactions. Pure $\mathrm{CH}$ spectra show a broad peak of $-\mathrm{NH}$ and $-\mathrm{OH}$ stretching vibration at $3300 \mathrm{~cm}^{-1}$, glycosidic bonds (C-O-C) characteristic of $\mathrm{CH}$ structure are seen at $1150 \mathrm{~cm}^{-1}$ and $1084 \mathrm{~cm}^{-1}$. Alamar Blue assay performed to assess the biocompatibility (Fig. 4) depicts higher viability for membranes synthesised with low molecular weight $\mathrm{CH}: \mathrm{HA}$ prepared with a ratio of 30:70.

\subsection{Figures}

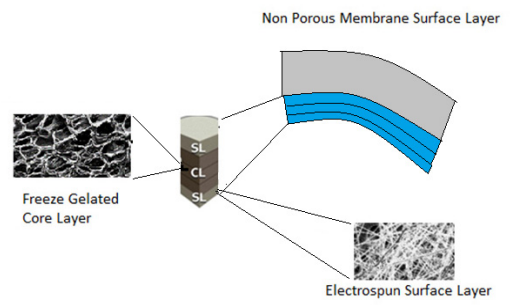

Figure 1. A schematic of a functionally graded guided tissue regenerative membrane for periodontal regeneration with surface and core layers of different morphologies.
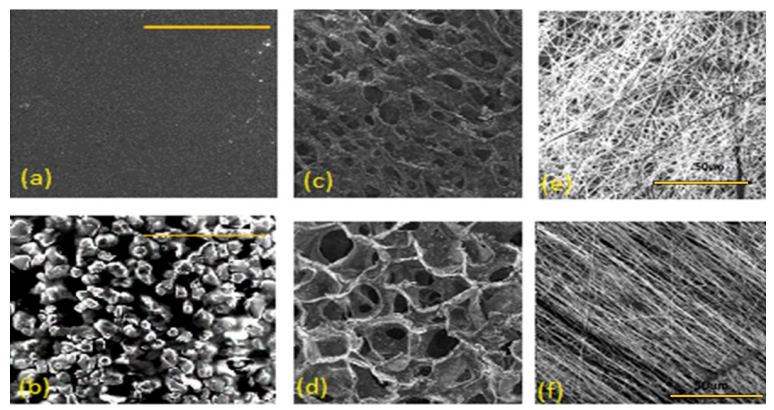

Figure 2(a, b). Top, bottom surface of solvent casted membranes; (c, d). Top, cross section of freeze gelated scaffolds; $(\mathbf{e}, \mathbf{f})$. Electrospun random and aligned fibres.

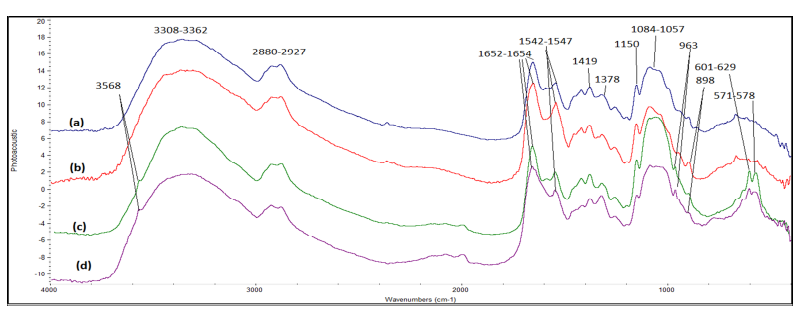

Figure 3. FTIR spectral data for freeze gelated: (a). $\mathrm{CH}$ in Ascorbic acid; (b). $\mathrm{CH}$ in Acetic acid; (c). $\mathrm{CH}$ in Acetic acid 50:50 HA; (d). $\mathrm{CH}$ in Ascorbic acid 50:50 HA.

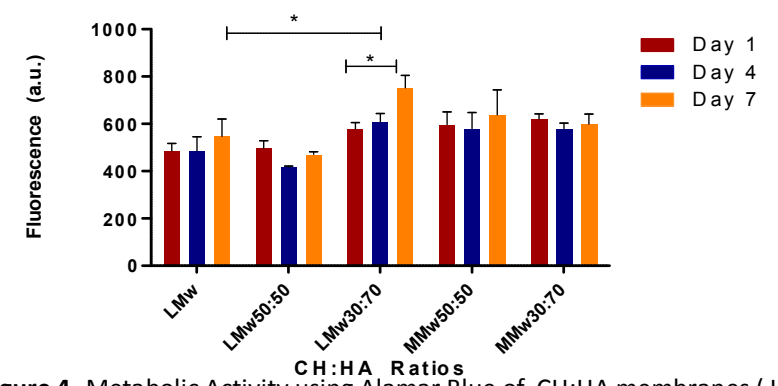

Figure 4. Metabolic Activity using Alamar Blue of $\mathrm{CH}: \mathrm{HA}$ membranes ( $\mathrm{LMw}$ 100:0, 70:30, , 50:50, 30:70 and MMw 50:50, MMw 30:70) with hES-MP's over a period of day 1, 4 and 7. Results shown are mean $\pm S D(n=3)$.

\section{CONCLUSIONS}

This study has described the fabrication of three different scaffolds with different morphologies and depicts the presence of bioactive and bioinert sides of non-porous membrane. The porous freeze gelated scaffolds were synthesised with energy and time efficient technique of freeze gelation. Electrospun fibres were obtained by using non-toxic solvents. Favourable biological response from the scaffolds with human osteosarcoma and mesenchymal progenitor cell line has suggested that these scaffolds can be used for periodontal tissue regeneration.

\section{ACKNOWLEDGEMENTS}

The authors acknowledge the support of ESPRC Delivery plan Pump Priming Grant.

\section{REFERENCES}

1. Grant MM. What do 'omic Technologies Have to Offer Periodontal Clinical Practice in the Future? Journal of Periodontal Research. 2012;47(1):2-14.

2. Bottino MC, Thomas V, Schmidt G, Vohra YK, Chu TM, Kowolik MJ, et al. Recent Advances in the Development of GTR/GBR Membranes for Periodontal Regeneration--a Materials Perspective. Dent Mater. 2012 Jul;28(7):703-21.

3. Hughes FJ, Ghuman M, Talal A. Periodontal Regeneration: a Challenge for the Tissue Engineer? Proc. Inst. Mech. Eng. H. 2010 Dec;224(12):1345-58. 